\title{
Emotional intelligence assessment in a graduate entry medical school curriculum
}

\author{
Eva M Doherty ${ }^{1 *}$, Patricia A Cronin ${ }^{2}$ and Gozie Offiah²
}

\begin{abstract}
Background: The management of emotions in the workplace is a skill related to the ability to demonstrate empathic behaviour towards patients; to manage emotional reactions in oneself and to lead others as part of a team. This ability has been defined as emotional intelligence (EI) and doctor's El may be related to communication skills and to patient satisfaction levels. This study reports on the use of two assessments of El as part of a course on Personal and Professional Development (PPD) in a graduate medical school curriculum.

Methods: Fifty one graduate entry medical students completed an eight session course on PPD between December 2005 and January 2006. Students completed two measures of El: self-report (EQ-i) and ability (MSCEIT V2.0) over a two year study period. The data gathered were used to explore the relationship between self-report and ability El and between El and student demographics, academic performance and change over time.

Results: Analysis of the El data demonstrated that self-report El did not change over time and was not related to ability El. Females scored higher than males on a number of self-report and ability El scores. Self-reported self-awareness was found to deteriorate in males and females over time. High self-reported El was found to be associated with poor performance on clinical competency assessments but with good performance on a number of bio-medical knowledge based assessments.
\end{abstract}

Conclusions: This report concludes that assessments of El can be incorporated into a medical school curriculum as part of a PPD programme and that the concept of El may be associated with performance in medical school.

Keywords: Emotional intelligence (EI), Empathy, Gender, Curriculum

\section{Background}

Emotional intelligence (EI) is recognised to be an important component of the doctor-patient relationship and has been demonstrated to be related to the level of trust and satisfaction felt by the patient towards the doctor [1-3]. The ability to demonstrate empathy is recognised to be an important emotional skill and studies of empathy in the medical student/trainee indicate that empathy deteriorates over the course of training and work [4-7]. Personality, stress, sleep deprivation, depression and burnout are just some of the possible mediating factors [8-11]. Medical educators have a responsibility to provide training for students to develop the ability to better manage their emotional responses to stressors and to prevent the attrition of empathy skills. A recent

\footnotetext{
* Correspondence: edoherty@rcsi.ie

${ }^{1}$ National Surgical Training Centre \& Graduate Medical School, Royal College of Surgeons in Ireland, 121, St Stephens Green, Dublin 2, Ireland

Full list of author information is available at the end of the article
}

systematic review sought to investigate whether such training programmes existed and if they were effective. The authors identified 26 studies which focussed on empathy skills training and demonstrated positive outcomes [12]. Similarly, several studies report the effectiveness of stressreduction programmes for medical students [13-15]. Thus the evidence indicates that interventions can be successful in reducing the effects of stress and in enhancing medical students'/ junior doctors' empathy skills.

The concept of EI has been incorporated into certain interpersonal skills sections of the Australian medical aptitudes test [16] and the evidence regarding EI and internationally recognised medical competencies has been recently systematically reviewed [17]. EI has been identified as one of several important concepts that could help move the culture of medical education ahead by creating a better learning, working and caring environment [18]. The evidence that EI may be a factor in 
medical competency is indicated by a recent study which found that self-rated emotional functioning and ability scores were found to be associated with scores on assessments of communication and physical examination skills in a sample of graduate medical students, [19] and studies of non-medical third level students have proposed that EI may be related to academic ability [20-22]. EI has been shown to be higher in female medical students at entry to medical school $[23,24]$ but to deteriorate over the course of medical training [25]. While a number of studies report on the use of EI in medical schools, only one study could be found which attempted to incorporate EI self-assessment and training into the medical curriculum [26]. The study sub-contracted an external agency to run the training course and although an increase in EI scores was demonstrated following attendance, only $34 \%$ of the cohort attended the complete course. Another study conducted at Peninsula graduate medical school UK, reported that the majority of students welcomed the opportunity to learn about EI and their self-rated emotional competencies [27] however very few studies to date have reported on how to design training programmes to provide students with the opportunity to learn explicitly about their own EI and such training programmes are well established in the business world $[28,29]$.

Finally, the majority of studies of medical students to date have used self-report measures of EI however recently it has been shown that it is possible to use an ability measure of EI in a medical school [30] although it is likely that self-report and ability measures may be measures of different aspects of EI [31].

\section{What is El?}

EI has been defined both as an ability and a trait and as a mixture of both. The ability definition describes EI as the ability to monitor one's own and others' feelings and emotions, to discriminate among them and to use this information to guide one's thinking and actions [32]. Trait theorists define EI as a constellation of emotionrelated self-perceptions and dispositions, assessed through self-report [33]. Mixed model theories define EI as an array of non-cognitive capabilities, competencies and skills [34].

\section{Measurement of El}

The trait models and mixed-models advocate the use of self-report as their mechanism of measurement, inferring that individuals who state that they function at various levels actually do. Examples of these models include the Emotional Quotient Inventory (EQ-i: [34]), the Emotional Competence Inventory (ECI: [35]), the Emotional Intelligence Scale (EIS: [36]) and finally the Trait Emotional Intelligence Questionnaire (TeiQue: [33]).
The Mayer-Salovey-Caruso Emotional Intelligence Test (MSCEIT V2.0: [37]) is an ability measure and assesses the four branches of Mayer and Salovey's EI ability model $[38,39]$. The MSCEIT V2.0 yields a profile of scores describing an individual's ability to perceive, use, understand and manage emotions. The topic of EI in psychology is a relatively new and controversial one and one of the difficulties is that self-report measures of EI do not correlate with ability measures of EI $[40,41]$. Notwithstanding the uncertainty in the EI literature, the concept of EI nevertheless offers the medical educator an attractive tool for the facilitation and development of the so called "noncognitive" abilities of the medical student/trainee $[42,43]$.

This study investigated the following research questions in a sample of graduate medical students:

Does EI change over the course of medical training?

Is there a gender difference in EI?

Are self-report EI scores associated with ability EI scores?

Is there a relationship between EI scores and academic achievement?

\section{Methods}

The first EI assessment (T1) was administered as part of a PPD course which took place between October and December 2006. The second EI assessment (T2) took place between the months of January and May 2008 when the students were in their second year of graduate medical programme. In the first year of the graduate medical programme, students' complete 12 modules encompassing systems based approach to medicine. The PPD course was given as part of one of these modules Health Behaviour and Society module. Permission to conduct the study and to analyse student EI data was granted by the Research Ethics Committee at the Royal College of Surgeons in Ireland.

Fifty one graduate entry medical students attended an eight week course on PPD. Each week, teaching took place during one three hour session. Student assessment was by means of two written assignments which counted for $10 \%$ of the final mark in the Health Behaviour and Society module, one of six modules in the first semester of the first year of the medical curriculum. In addition, students maintained an eportfolio worth $20 \%$ of the final mark in the Clinical Competencies module. Students also completed other PPD related activities as part of the standard curriculum in their first year such as team projects, integrated clinical communication skills practice and clinical placements. The topics in the eight week PPD course were as follows:

1. Professionalism and the eportfolio (two sessions).

2. Stress and stress management (three sessions). 
3. Leadership and emotional intelligence (three sessions).

Teaching methods incorporated didactic presentations, small and large group activities and discussion. (See Additional file 1 for an outline of content and teaching methods). Self-report EI was assessed as part of the PPD course at T1. Two years later (T2), students were requested to complete the self-report EI once more and an ability EI measure. Only the EI component of the programme and the results of the analysis of the EI data will be described.

\section{El components of the PPD programme}

EI was presented to the class as an important component of communication and leadership abilities and selfcare. The first session, Leadership one, consisted of a slide presentation and discussion. Students subsequently received instructions on how to complete the EQ-i selfreport measure online and assured of confidentiality. Students' permission to publish annonymised results was requested in writing. Only the principal author (ED) had access to EI profiles in addition to the students.

Prior to the second session (Leadership two) each student completed the online EI assessment and received an individualised resource report from the principal lecturer; a practising Clinical Psychologist (ED). Group feedback was provided during the Leadership two session and students were invited to contact the lecturer for individual feedback if desired. The third session on leadership was designed to demonstrate how the EI profiles were applicable to various aspects of their professional roles in the future as practitioners and leaders with tips for developing EI competencies.

\section{Student assessment}

Assignment two of the PPD module required the student to study their individual EI profiles and conduct a SWOT (Strengths, Weaknesses, Opportunities and Threats) analysis. The aim of the assignment was to facilitate students' reflection on their EI profile and on strategies to enhance emotional awareness and understanding.

\section{Description of El measures used in PPD course Emotional Quotient Inventory (EQ-i)}

Two measures of EI were used. The first was the EQ-i [34]; a self-report measure completed online through a website (www.mhsassessments.com) although a pen and paper version is available. The measure comprises of either 133 or 125 brief items, employing a five-point response set (ranging from "Not true of me" to "True of me") and takes approximately 30-40 minutes to complete with no imposed time limits. The EQ-i renders four validity scale scores, a total EQ score, five composite scale scores and 15 EQ subscale scores. EQ-i raw scores are converted into standard scores based on a mean of 100 and a standard deviation of 15 (similar to IQ scores). This score conversion allows for comparison with the normative group and also with other EI measures using standard scores such as the second measure used in this study.

Internal consistency scores for the EQ-i (Cronbachs alpha) range from $r=.69$ (Social Responsibility) to $r=.86$ (Self-Regard) with an overall average coefficient of $r=.76$ for the full scale score. Second-order confirmatory factorial analysis provides support for the five composite scales described in the test manual. Correlations coefficients ranged from $r=.30$ to $r=.70$.

The authors conclude that these results support the tenet that the EQ-i subscales are measuring the constructs that they were intended to measure but are not so high as to suggest that the EQ-i is a duplication of existing inventories. Other forms of validity are presented in detail in the manual. A revised edition of the EQ-i has been made available by the agency since June 2011 and a $360^{\circ}$ administration is also available. Figure 1 shows the subscales of the EQ-i and a brief description.

\section{Mayer-Salovey-Caruso emotional intelligence test (MSCEIT V2.0)}

The MSCEIT (pronounced "Mes-keet"), is an ability-based instrument which measures how well people perform emotional tasks and solve problems involving emotional situations [37]. In some subscales, the test presents a series of photos and pictures and the respondent is required to identify the emotions portrayed. Other subscales ask the respondent to choose the most appropriate strategy to solve an interpersonal situation. The measure is distributed by the same agency in the same way as the EQ- $\mathrm{i}$ described previously (www.mhsassessments.com). There is a paper and pen version available however online administration is the usual method. The measure comprises of 141 test items and takes between 30-45 minutes to complete. The main scores that the MSCEIT V2.0 produces are an overall total EIQ score, two area EIQ scores, four branch EIQ scores and eight task scores. There are also three supplemental scores: a scatter score, a positivenegative bias score and an omission rate. Reliability coefficients for the full scale score are quoted in the manual and demonstrate good internal consistency for the full scale score $(r=.91)$ with subscale scores ranging from $r=.74$ to $r=.89$. Good evidence for face, content, factorial and predictive validity is presented in the manual. Figure 2 gives the subscales of the MSCEIT with a brief description.

\section{Results and discussion \\ Statistical analysis}

Data were analysed using SPSS version 18.0. As the data were normally distributed, independent sample t-tests 


\section{Total EQ-i}

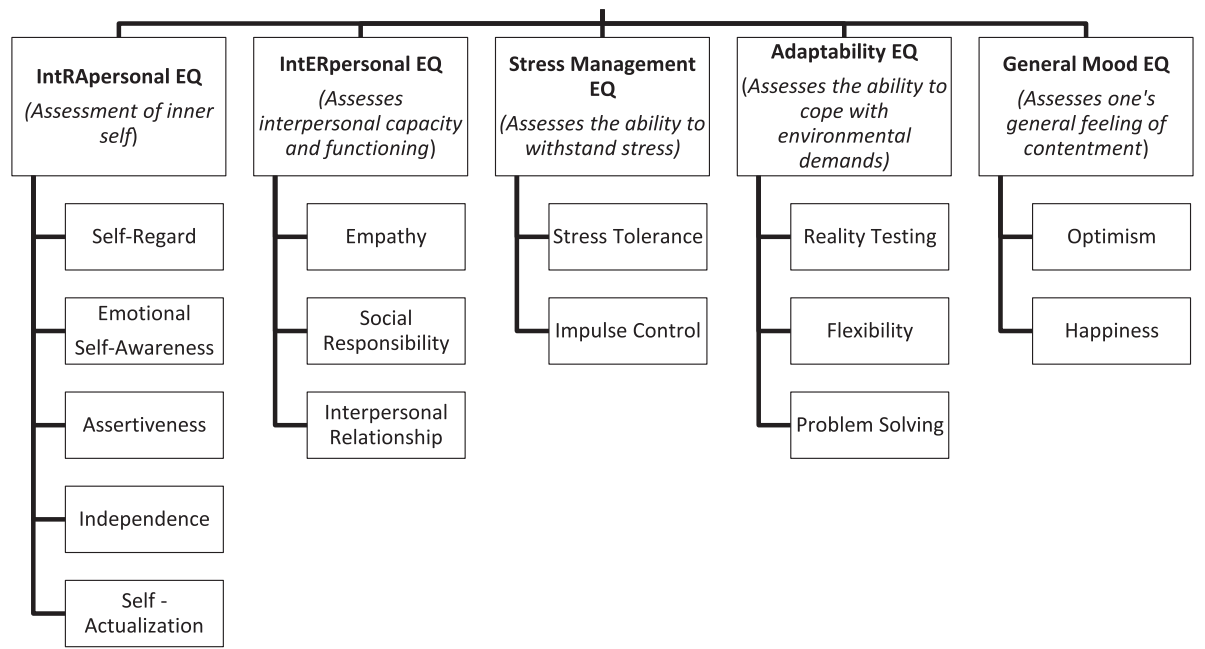

Figure 1 Subscales of the EQ-i and a brief description.

were conducted to compare the means of EQ-i and MSCEIT V2.0 scales with regards to gender and age. The comparison of means between the students' scores at first assessment and at follow up was conducted using the paired t-test. Pearson's product-moment correlation was used to explore the relationship between continuous variables.

\section{Respondents}

Forty nine $(98 \%)$ of the 51 students in the class completed the EQ-i measure at T1 and gave consent for

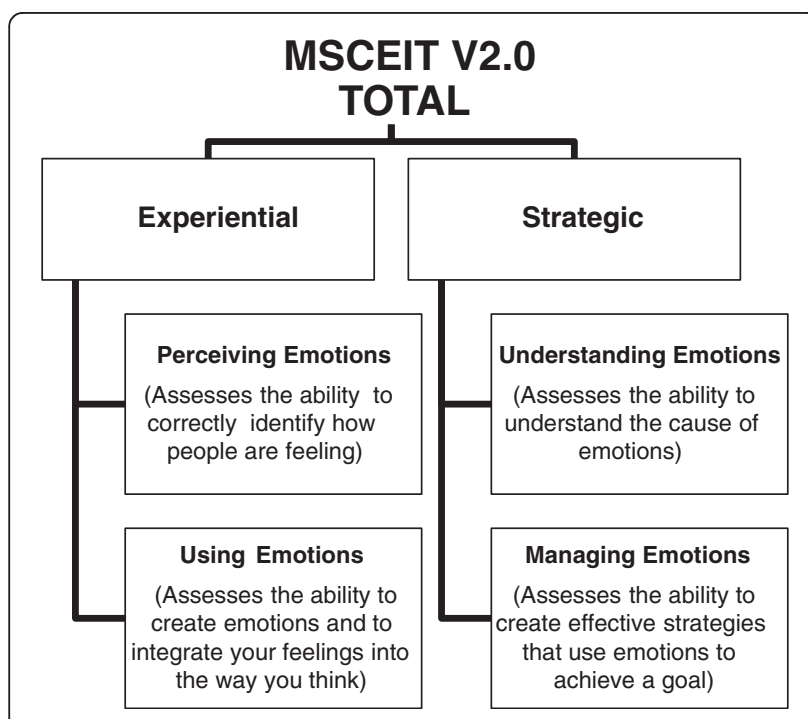

Figure 2 Subscales of the MSCEIT V2.0 and a brief description. their anonymised data to be analysed and results published. At T2, thirty three (67\%) students completed the EQ-i and the MSCEIT V2.0. The mean EQ-i at T1 was 105.6 (range 73 - 131). Differences between mean EQ-i scores and proportion of males/females, ages, were investigated using t-tests and chi-square analysis and none were found (Table 1). However, Pearsons correlation coefficient indicated that student age was significantly positively associated with EQ-i scores $(\mathrm{r}=.36, \mathrm{p} \leq .05)$ but not with MSCEIT V2.0 scores. There was no difference found between the EQ-i scores of those students at T1 who did not complete the second EI assessment and the EQ-i scores of the students who did.

The following questions were investigated:

\section{Does El change over the course of medical training?}

There was no significant difference in self-report total EQ-i scores from T1 to T2. In subscale analysis however, there was a significant decrease in EQ-i Self-Awareness scores: $(M=107.7, \mathrm{SD}=15.9)$ versus $(\mathrm{M}=103.8, \mathrm{SD}=13.9$, $\mathrm{p} \leq .05)$. The mean difference in the EQ-i Self-Awareness scores was 3.91 points with a $95 \%$ confidence interval ranging from 0.254 to 7.564 . The eta-squared statistic (.13) indicated a moderate to large effect size.

Scores on one of the self-report EQ-i subscales (Reality Testing) for males increased significantly from T1 to T2. $(\mathrm{M}=98.9, \mathrm{SD}=8.9)$ versus $(\mathrm{M}=106.8, \mathrm{SD}=13.7, \mathrm{p} \leq .05)$; while scores on the interpersonal skills section, a section of the EQ-i comprising of three subscales (Empathy, Social Responsibility and Interpersonal Relationship) deteriorated between $\mathrm{T} 1$ and $\mathrm{T} 2$ in females $(\mathrm{M}=113.2, \mathrm{SD}=10.7)$ versus $(M=104.6, \mathrm{SD}=10.2, \mathrm{p} \leq .01)$. 
Table 1 Sample characteristics at first (T1) and second (T2) assessment: sex, mean ages (standard deviation), mean El (standard deviation)

\begin{tabular}{|c|c|c|c|c|c|c|c|c|}
\hline & & $\mathrm{T} 1$ & & & & & & \\
\hline & & $n=49$ & & & & & & \\
\hline Sex & $\mathbf{N}$ & Age & EQ-i & Sex & $\mathrm{N}$ & Age & EQ-i & MSCEITV2.0 \\
\hline Female & 31 & 24.03(1.65) & 105.87(10.17) & Female & 18 & $26.28(1.67)$ & 109.33(11.22) & $107.47(13.12)$ \\
\hline Male & 18 & 23.82(2.01) & $104.35(9.76)$ & Male & 15 & $25.71(1.77)$ & $104.33(12.75)$ & $101.04(15.51)$ \\
\hline
\end{tabular}

Note: All means non significantly different.

\section{Is there a gender difference in EI?}

While no significant difference was found on total EQ-i scores between male and female students, differences were found in favour of females on some of the subscale scores. Comparison of means indicated that female students scored significantly higher than males on selfreported empathy $(M=110.6, S D=11.4)$ versus $(M=99.3$, $\mathrm{SD}=12.9, \mathrm{p} \leq .01)$ and social responsibility $(\mathrm{M}=107.1$, $\mathrm{SD}=10.4)$ versus $(\mathrm{M}=97.2, \mathrm{SD}=16.4, \mathrm{p} \leq .01)$ (Figure 3$)$.

In the analysis of EQ-i scores at T2, no further differences in male versus female self-reported total EQ-i scores were found. Female students maintained their significantly higher empathy and social responsibility scores at the second time point.

With regard to the ability measure of EI, female students scored significantly higher than the male students on the Strategic EIQ area, $(M=108.8, S D=10.4)$ versus $(\mathrm{M}=100.3, \mathrm{SD}=10.9, \mathrm{p} \leq .05)$ (Figure 4).

\section{Are self-report El scores associated with ability El scores?}

To investigate if there was a statistically significant association between self-reported EI scores (as measured by the EQ-i) and ability EI scores (as measured by the MSCEIT V2.0), a Pearson-product moment correlation was computed and was not significant.

\section{Is there a relationship between El scores and academic achievement?}

Scores on all module assessments were compared to EQ-i scores at T1 using Pearson's correlation coefficient. EQ-i scores were negatively associated with performance on the Clinical Competency (CC) modules $(r=-.38$,

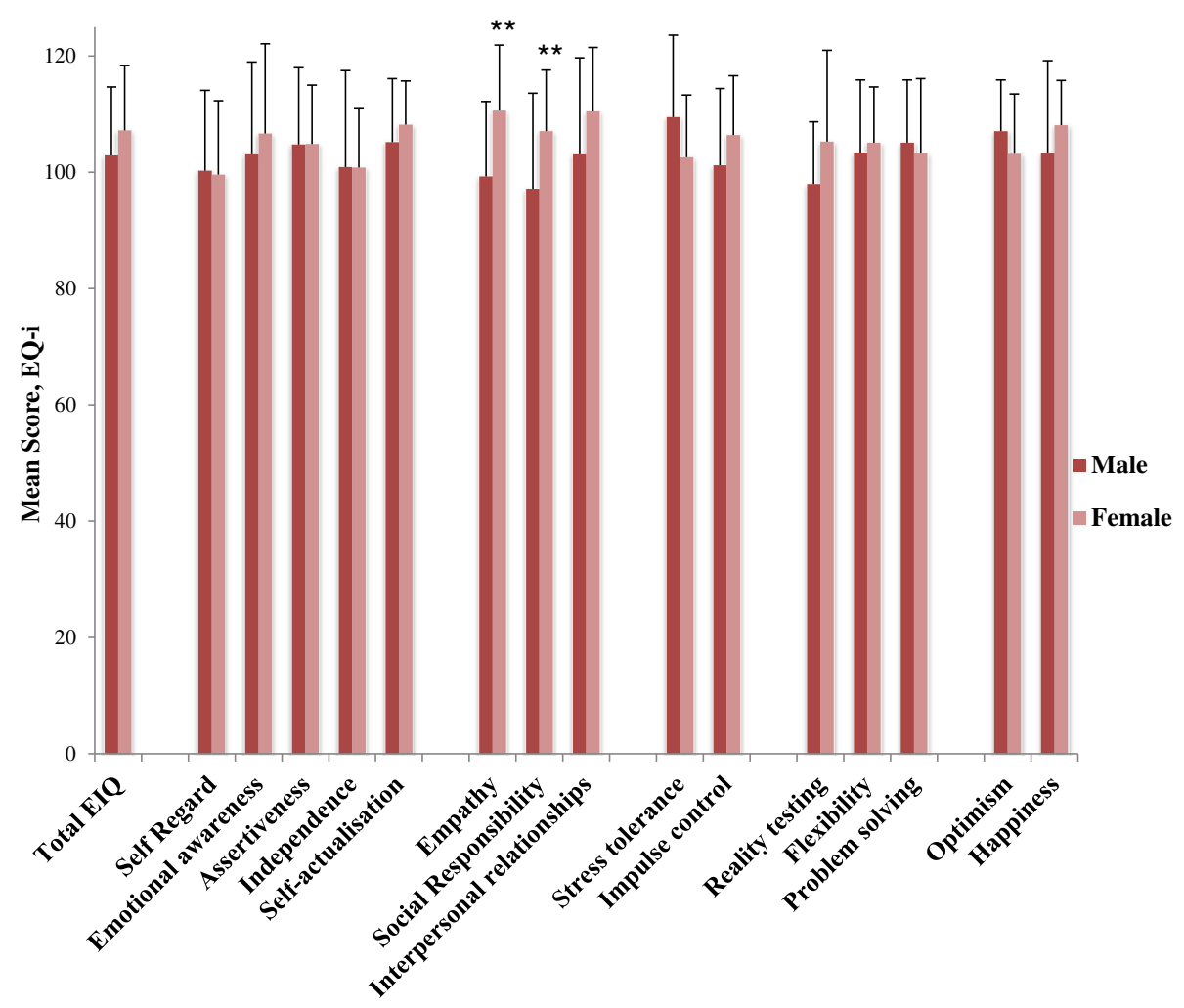

Figure 3 Comparison of self-reported emotional intelligence scores (EQ-i) according to gender at the first assessment (T1). 


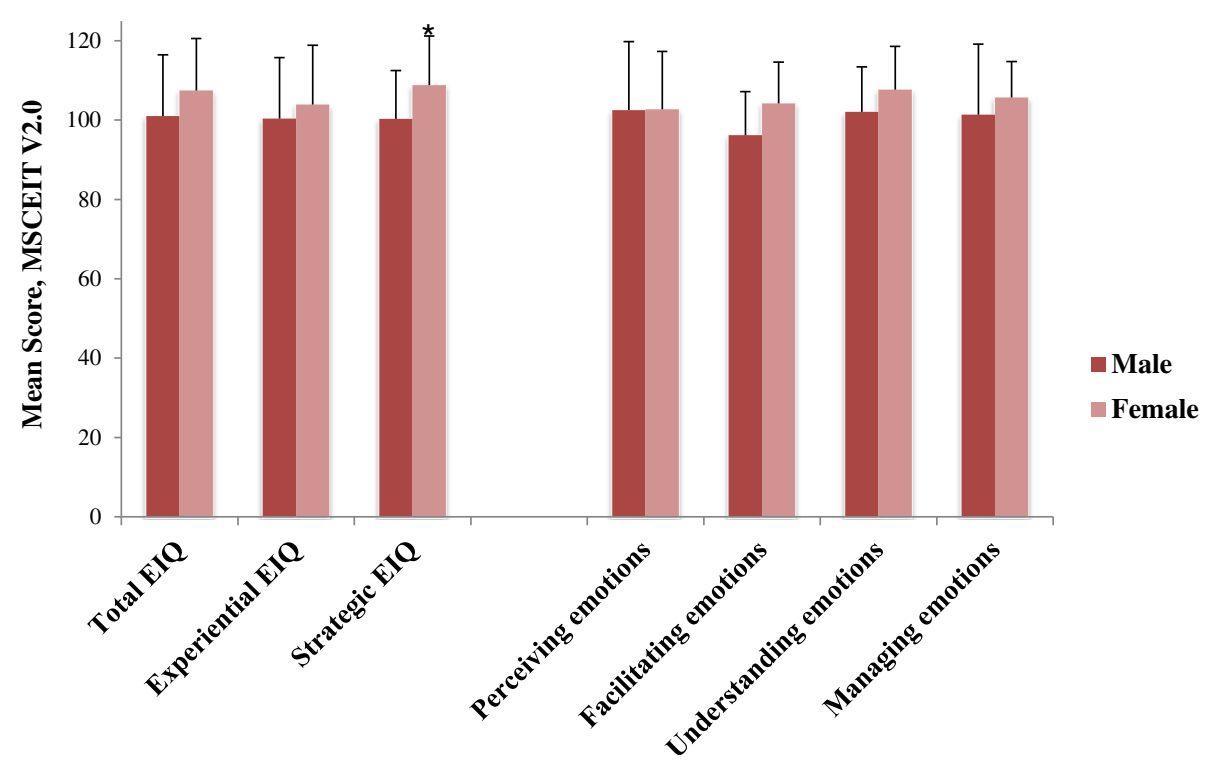

Figure 4 Comparison of ability emotional intelligence scores (MSCEIT V2.0) according to gender at the second assessment (T2).

$\mathrm{p} \leq .01)$ such that students who rated themselves as possessing good emotional competencies were more likely to obtain low scores on these assessments. These modules are assessed by means of OSCE (Objective Structured Clinical Examinations), designed to assess both clinical and communication skills. Conversely, scores on another EQ-i subscale (Impulse Control) were found to be positively associated with scores on four other module assessments; Health Behaviour and Society, Evidence Based Health, Haemopoietic and Immune Systems, Genitourinary and Endocrine (all r's between .28 and .50).

Correlation coefficient scores for EQ-i, MSCEIT V2.0 and academic performance were compared at T2. Once again an association between high EQ-i scores and poor performance on the $\mathrm{CC}$ module were found with medium negative correlations demonstrated between two of the EQ-i subscale scores and OSCE scores (Inter-personal Relationship: $\mathrm{r}=-.39, \mathrm{p} \leq .05$; Self-Regard: $\mathrm{r}=-.39$, $\mathrm{p} \leq .05)$. Similarly, medium negative correlations were also found between two EQ-i subscales (Interpersonal relationship: $\mathrm{r}=-.39, \mathrm{p} \leq .05$; Happiness: $\mathrm{r}=-.35, \mathrm{p} \leq .05)$ and performance on one of the other modules, the Biology and Epidemiology Disease module. These results suggest that students who rate themselves as happier with good interpersonal relationships and high self-regard were more likely to obtain lower marks on these assessments.

\section{Conclusions}

The purpose of this study was to demonstrate that the concept of EI can be included in a PPD course within the context of a systems based medical curriculum. This represents a first step for this graduate medical school in addressing the difficult topic of professionalism and the mechanisms, which can be used to teach and assess it. The EI data gathered within the context of the course activities were statistically analysed and a number of significant associations between EI scores and gender, time and academic assessments were identified.

The finding that both male and female students rated their self-awareness competency at a lower level at T2 compared to $\mathrm{T} 1$ is in agreement with the evidence discussed above that empathy skills deteriorates over the course of medical training. Female students demonstrated superior emotional competencies on some of the self-report and ability EI scores. However females were also more likely to report deterioration in some of these emotional competencies over time. It may be that the medical education environment encourages females to adapt their interpersonal skills and emotional competencies to become more similar to their male counterparts.

A number of self-report EI competencies were associated negatively with performance on some of the academic modules and this warrants further investigation. There is evidence that self-rated EI is closely associated with personality $[33,41]$ and so this may be a reflection of the nature of the relationship between personality and academic performance in medical training [44]. The finding that those students who rated themselves highly on certain emotional competencies were also more likely to obtain low scores on assessments of their clinical and communication skills may be an indication of their lack of insight into their true abilities or may be to do with the validity of the assessments themselves. An inverse relationship between certain self-report EI competencies and performance on clinical OSCEs was identified again at T2 and this supports the possible association between 
lack of insight and poor clinical performance. In contrast, strong to medium positive correlations between the EQ-i subscale Impulse Control and four module scores were demonstrated at $\mathrm{T} 1$ such that a perceived high ability to manage frustration was associated with high scores on these knowledge based assessments. However at T2, an inverse association between certain selfreport EI competencies and performance on one of the knowledge based modules was found which indicates that the relationship between self-reported EI and actual academic performance is not straightforward and should be investigated further.

Ability EI and self-report EI did not correlate and there were no associations between the ability EI scores and academic performance indicating once more that these two conceptualisations of EI are not assessing the same aspect of emotional competency. The sample size at T2 which compared the ability EI scores with academic performance was small $(n=33)$ and so the analysis was vulnerable to a Type II error (i.e. failure to find an association when one exists).

There are a number of limitations to this study. The analysis was conducted within the practical limitations necessarily encountered within the confines of the demands of a medical curriculum. The study thus took advantage of the availability of the EI data to explore pertinent questions concerning EI and medical students against performance. The findings are very preliminary and will require replication in a more methodologically robust study. The size of the samples at both assessment points were small and in particular at T2. Finally, a bonferroni correction was not applied and so it is possible that some of the significant correlations occurred by chance. Replication with more participants is required to investigate the relationships identified.

Though a very small cohort, our study has shown that there are significant changes in several subscales of the self-reported and ability scores of EI and the subsequent correlations with academic performance. We have also shown that it is possible to include student EI assessment in a PPD programme within a medical curriculum. We thus invite other medical educators to adapt this PPD programme including EI assessment as outlined to suit their own needs and to evaluate it within the context of their medical curricula.

\section{Additional file}

Additional file 1: An outline of the Personal and Professional Development course.

\section{Competing interests}

The authors declare that they have no competing interests.

\section{Authors' contributions}

ED carried out the design of the study and acquisition of data. ED also collated the analysis and interpretation of the data and the drafting of the manuscript. PC participated in the analysis of data and in drafting the manuscript. GO was involved in revising the manuscript critically. GO was also involved in preparing the tables and figures as well as in preparing and analysing some of the data. All authors read and approved the final manuscript.

\section{Authors' information}

ED is Director of Human Factors and Patient Safety at the National Surgical Training Centre at the Royal College of Surgeons in Ireland (RCSI). Prior to this appointment in 2009, Eva was Senior Lecturer/Clinical Psychologist at the Graduate School of Medicine at RCSI.

PC was a clinical tutor in 2009 on the graduate entry medical programme with the Royal College of Surgeons in Ireland. She is also a specialist registrar in general surgery.

GO is a surgical trainee and in 2010 was the clinical tutor at the Graduate entry medical school in Royal College of Surgeons in Ireland. She has recently taken up a lecturer post in the Human Factors programme at the National Surgical Training Centre in Royal College of Surgeons in Ireland.

\section{Acknowledgements}

The authors would like to thank the class of 2010, the first class to graduate from the new graduate medical school at RCSI, for participating in this project and for their tolerance of their teachers' curricular experiments! We wish them every success in their medical careers. Thanks also to Professor Alan Johnson, Director of the Graduate Medical School at the time for his enduring support and enthusiasm for the project.

\section{Author details}

${ }^{1}$ National Surgical Training Centre \& Graduate Medical School, Royal College of Surgeons in Ireland, 121, St Stephens Green, Dublin 2, Ireland. ${ }^{2}$ Graduate Medical School, Royal College of Surgeons in Ireland, Ballymoss Road, Sandyford Industrial Estate, Dublin 18, Ireland.

Received: 20 November 2012 Accepted: 4 March 2013

Published: 7 March 2013

\section{References}

1. Wagner PJ, Moseley GC, Grant MM, Gore JR, Owens C: Physicians' emotional intelligence and patient satisfaction. Fam Med 2002, 34(10):750-754.

2. Weng HC: Does the physician's emotional intelligence matter? Impacts of the physician's emotional intelligence on the trust, patient-physician relationship, and satisfaction. Health Care Manage Rev 2008, 33(4):280-288.

3. Weng HC, Chen HC, Chen HJ, Lu K, Hung SY: Doctors' emotional intelligence and the patient-doctor relationship. Med Educ 2008 42(7):703-711

4. Hojat M, Mangione S, Nasca TJ, Rattner S, Erdmann JB, Gonnella JS, Magee M: An empirical study of decline in empathy in medical school. Med EduC 2004, 38(9):934-941.

5. Hojat M, Vergare MJ, Maxwell K, Brainard G, Herrine SK, Isenberg GA, Veloski J, Gonnella JS: The devil is in the third year: a longitudinal study of erosion of empathy in medical school. Acad Med 2009, 84(9):1182-1191.

6. Newton BW, Barber L, Clardy J, Cleveland E, O'Sullivan P: Is there hardening of the heart during medical school? Acad Med 2008, 83(3):244-249.

7. Shapiro J: Perspective: does medical education promote professional alexithymia? A call for attending to the emotions of patients and self in medical training. Acad Med 2011, 86(3):326-332.

8. Rosen IM, Gimotty PA, Shea JA, Bellini LM: Evolution of sleep quantity, sleep deprivation, mood disturbances, empathy, and burnout among interns. Acad Med 2006, 81(1):82-85.

9. McManus IC, Keeling A, Paice E: Stress, burnout and doctors' attitudes to work are determined by personality and learning style: a twelve year longitudinal study of UK medical graduates. BMC Med 2004, 2:29.

10. West CP, Tan AD, Habermann TM, Sloan JA, Shanafelt TD: Association of resident fatigue and distress with perceived medical errors. JAMA 2009, 302(12):1294-1300

11. Dyrbye LN, Massie FS Jr, Eacker A, Harper W, Power D, Durning SJ, Thomas MR, Moutier C, Satele D, Sloan J, et al: Relationship between burnout and 
professional conduct and attitudes among US medical students. JAMA 2010, 304(11):1173-1180.

12. Satterfield JM, Hughes E: Emotion skills training for medical students: a systematic review. Med Educ 2007, 41(10):935-941.

13. Finkelstein C, Brownstein A, Scott C, Lan YL: Anxiety and stress reduction in medical education: an intervention. Med Educ 2007, 41(3):258-264.

14. Krasner MS, Epstein RM, Beckman H, Suchman AL, Chapman B, Mooney CJ, Quill TE: Association of an educational program in mindful communication with burnout, empathy, and attitudes among primary care physicians. JAMA 2009, 302(12):1284-1293.

15. Shapiro SL, Astin JA, Bishop SR, Cordova M: Mindfullness-based stress reduction for health care professionals:results from a randomized trial. Int J Stress Manag 2005, 12:164-176.

16. Carr SE: Emotional intelligence in medical students: does it correlate with selection measures? Med Educ 2009, 43(11):1069-1077.

17. Arora S, Ashrafian H, Davis R, Athanasiou T, Darzi A, Sevdalis N: Emotional intelligence in medicine: a systematic review through the context of the ACGME competencies. Med Educ 2010, 44(8):749-764.

18. Grewal D, Davidson HA: Emotional intelligence and graduate medical education. JAMA 2008, 300(10):1200-1202.

19. Stratton TD, Elam CL, Murphy-Spencer AE, Quinlivan SL: Emotional intelligence and clinical skills: preliminary results from a comprehensive clinical performance examination. Acad Med 2005, 80(10 Suppl):S34-S37.

20. Barchard KA: Does emotional intelligence assist in the prediction of academic success? Ed Psych Meas 2003, 63:50840-50858.

21. Romanelli F, Cain J, Smith KM: Emotional intelligence as a predictor of academic and/or professional success. Am J Pharm Educ 2006, 70(3):69.

22. Parker JDA, Summerfeldt LJ, Hogan MJ, Majeski SA: Emotional intelligence and academic success: examining the transition from highschool to university. Personal Individ Differ 2004, 36:163-172.

23. Austin EJ, Evans P, Goldwater RV, Potter V: A preliminary study of emotional intelligence,empathy and exam performance in first year medical students. Pers and Ind Diffs 2005, 39:1395-1405.

24. Carrothers RM, Gregory SW Jr, Gallagher TJ: Measuring emotional intelligence of medical school applicants. Acad Med 2000, 75(5):456-463.

25. Stratton TD, Saunders JA, Elam CL: Changes in medical students' emotional intelligence: an exploratory study. Teach Learn Med 2008, 20(3):279-284

26. Fletcher I, Leadbetter P, Curran A, O'Sullivan H: A pilot study assessing emotional intelligence training and communication skills with 3rd year medical students. Patient Educ Couns 2009, 76(3):376-379.

27. Lewis $N$, Rees $C$, Hudson N: Helping medical students identify their emotional intelligence. Med Educ 2004, 38(5):563.

28. Kunnanatt JT: Emotional intelligence:the new science of interpersonal effectiveness. Hum Resour Quar 2004, 15:489-495.

29. Lindebaum C: A critical examination of the relatiobship between emotional intelligence and transformational leadership. J Manage Stud 2010, 47:7

30. Todres M, Tsimtsiou Z, Stephenson A, Jones R: The emotional intelligence of medical students: an exploratory cross-sectional study. Med Teach 2010, 32(1):e42-e48

31. Brannick MT, Wahi MM, Arce M, Johnson HA, Nazian S, Goldin SB: Comparison of trait and ability measures of emotional intelligence in medical students. Med Educ 2009, 43(11):1062-1068.

32. Salovey P, Mayer J: Emotional intelligence. Imagination, cognition, and personality 1990, 9:185-211

33. Petrides KV, Furnham A: Trait emotional intelligence: behavioural validation in Two studies of emotion recognition and reactivity to mood induction. Eur J Personal 2003, 17:39-57.

34. Bar-On R: Bar-On Emotional Quotient Inventory: technical manual. Toronto Multi-Health Systems Toronto; 1997

35. Boyatzis RE, Goleman D, Rhee KS: Clustering competence in emotional intelligence. In The handbook of emotional intelligence: Theory development, and assessment, and application at home, school, and in the workplace San Francisco, CA. Edited by Bar-On R, Parker JDA. San Francisco, CA: Jossey-Bass; 2004.

36. Schutte NS, Malouff JM, Hall LE, Haggerty DJ, Cooper JT, Golden CJ, et al: Development and validation of a measure of emotional intelligence. Personality and Individual Differences. 1998:167-177.

37. Mayer JD, Salovey P, Caruso D: Mayer-Salovey-Caruso Emotional Intelligence Test (MSCEIT). Toronto: MHS Publ; 2002.
38. Mayer JD, Salovey P: The intelligence of emotional intelligence. Intelligence 1993, 17:433-442.

39. Mayer JD, Salovey P: What is emotional intelligence. In Emotional Development and Emotional Intelligence. New York: Basic Books; 1997.

40. Mayer JD, Roberts RD, Barsade SG: Human abilities: emotional intelligence. Annu Rev Psychol 2008, 59:507-536.

41. Roberts RD, Zeidner M, Matthews G: Does emotional intelligence meet traditional standards for an intelligence? Some new data and conclusions. Emotion 2001, 1(3):196-231.

42. Elam CL: Use of "emotional intelligence" as one measure of medical school applicants' non cognitive characteristics. Acad Med 2000, 75:445-446.

43. Taylor C, Farver C, Stroller JK: Can emotional intelligence training server as an alternative approach to teaching professionalism to residents? Acad Med 2011, 86:1551-1554

44. Doherty EM, Nugent E: Personality factors and medical training: a review of the literature. Med Educ 2011, 45(2):132-140.

doi:10.1186/1472-6920-13-38

Cite this article as: Doherty et al.: Emotional intelligence assessment in a graduate entry medical school curriculum. BMC Medical Education 2013 13:38.

\section{Submit your next manuscript to BioMed Central and take full advantage of:}

- Convenient online submission

- Thorough peer review

- No space constraints or color figure charges

- Immediate publication on acceptance

- Inclusion in PubMed, CAS, Scopus and Google Scholar

- Research which is freely available for redistribution

Submit your manuscript at www.biomedcentral.com/submit
C Biomed Central 